Análisis de la distribución del grisón (Galictis vittata)

(Carnivora: Mustelidae) en el Caribe colombiano

\title{
Analysis of the distribution of the Grison (Galictis vittata) (Carnivora: Mustelidae) in the Colombian Caribbean
}

\author{
J. Sebastián Jiménez-Alvarado ${ }^{1}$, Angélica Arias-Ocampo ${ }^{1,2}$, Alexandra Pineda-Guerrero ${ }^{1}$, Diego A. Zárrate-Charry ${ }^{1,3}$, \\ I. Mauricio Vela-Vargas ${ }^{1}$, Julio Chacón-Pacheco ${ }^{4}$ y José F. González Maya ${ }^{1 *}$
}

\begin{abstract}
1 Proyecto de Conservación de Aguas y Tierras, ProCAT Colombia/Internacional, Carrera 13 núm. 96-82 Oficina 205. Bogotá, Colombia. Email:jsjimeneza@uqvirtual.edu.co (JSJA), alexapin88@hotmail.com (APG), mauricio.vela@gmail.com (IMVV), ifgonzalezmaya@gmail.com (JFGM).

${ }_{2}^{2}$ Programa de Biología, Facultad de Ciencias Básicas, Universidad del Magdalena, Carrera 32 núm. 22 - 08, Santa Marta, Magdalena, Colombia. Email: ariasocampo.a@gmail.com (AAO).

${ }^{3}$ College of Forestry, Oregon State University, Corvallis 97331-5704. Oregon, EE.UU. Email: godiezcharry@gmail.com (DAZC).

${ }^{4}$ Biological Art, Montería, Córdoba, Colombia/Grupo de Biodiversidad Unicórdoba, Facultad de Ciencias Básicas, Departamento de Biología. Universidad de Córdoba, Carrera 6 núm. 76-103, Montería. Córdoba, Colombia. Email: jchacon_bio@hotmail.com (JCP).

* Autor de correspondencia
\end{abstract}

\begin{abstract}
The Grison (Galictis vittata) is a mustelid carnivore with a wide distribution in the American continent, including Colombia. However, is one of the less studied carnivore species in Colombia, with scarce information regarding distribution, ecology and conservation status. We compiled all records and observations available for the species in the Caribbean region of Colombia. Additionally, through field work held throughout the region, we present new confirmed records for the species. We obtained a total of 78 records, distributed in seven of the eight departments of the Caribbean Region of Colombia; of these, 54 records are new for the region and are located in five departments. The new records expand the known distribution of the species in $162 \mathrm{~km}$ to the South and $36,500 \mathrm{~km}^{2}$ in extent from its previously estimated distribution, also confirming its presence in three departments for the first time. Our results extend the knowledge of the species for the Caribbean region of Colombia by providing new records and data on distribution and extending its known range. These results also highlight the need for further study of the species regarding most aspects of its natural history.
\end{abstract}

El grisón (Galictis vittata) es un carnívoro mustélido con una amplia distribución en el continente americano, incluida Colombia. Sin embargo, se trata de una de las especies de carnívoros menos estudiada en Colombia, con déficit de información sobre su distribución, ecología y estado de conservación. Se recopilaron todos los registros y observaciones disponibles de la especie en la región del Caribe colombiano. Asimismo, a través del trabajo de campo realizado en toda la región, se presentan nuevos registros confirmados de la especie. Se obtuvo un total de 78 registros distribuidos en siete de los ocho departamentos de la Región Caribe de Colombia; de éstos, 54 son nuevos registros en la región y se encuentran en cinco departamentos. Los nuevos registros expanden la distribución conocida de la especie en $162 \mathrm{~km}$ lineales hacia el sur y en aproximadamente $36,500 \mathrm{~km}^{2}$ en extensión respecto de su distribución previamente estimada, y confirman su presencia en tres departamentos por primera vez. Los resultados amplían el conocimiento de la especie en el Caribe colombiano, y proporcionan nuevos registros y datos sobre la distribución, lo que amplía su distribución conocida. Dichos resultados también muestran la necesidad de estudiar la especie en cuanto a la mayoría de los aspectos de su historia natural.

Key words: Colombia; conservation; distribution; Grison; habitat.

๑ 2016 Asociación Mexicana de Mastozoología, www.mastozoologiamexicana.org

\section{Introducción}

El grisón (Galictis vittata; Shreber 1776) es una especie de la familia Mustelidae que se distribuye a lo largo de las áreas tropicales y subtropicales de América. Su distribución actual conocida abarca desde México, pasando por Centroamérica y Suramérica, hasta Paraguay, el norte de Argentina y el sur de Brasil (Yensen y Tarifa 2003; Cuarón et al. 2008). En Colombia, la especie se distribuye a lo largo del Caribe, los Andes y la Orinoquía (Solari et al. 2013), aunque existen menciones anteriores sin confirmarse en la Amazonía y el Pacífico (Alberico et al. 2000; Muñoz-Saba y Alberico 2004; AsprillaPerea et al. 2013). Si bien la especie presenta una amplia distribución tanto en el ámbito global como 
nacional, la información sobre su ecología, distribución y estado de conservación es escasa e imprecisa.

Hasta la fecha, no existe ningún estudio específico publicado sobre la especie en el país, y la información es principalmente de naturaleza anecdótica (Calderón-Capote et al. 2015). En el ámbito nacional, la especie ya ha sido previamente identificada entre las prioridades de investigación, principalmente en términos de su biología, ecología y distribución (González-Maya et al. 2011). En Colombia, el grisón se encuentra dentro del Apéndice III de la Convención sobre el Comercio internacional de Especies Amenazadas de Fauna y Flora Silvestres (CITES), y en el entorno internacional, según la Unión Internacional para la Conservación de la Naturaleza (IUCN), está catalogada como en Preocupación Menor (Cuarón et al. 2008). Sin embargo, en Colombia se desconoce su situación real de conservación, ya que, como se mencionó, no existe información suficiente para evaluar su estado actual (González-Maya et al. 2011).

En especial, en la región Caribe existen pocos especímenes depositados en colecciones, y la distribución de la especie propuesta en varias fuentes parece no estar corroborada por dichos registros (Alberico et al. 2000; Cuarón et al. 2008). En este trabajo se revisan todos los registros confirmados de Galictis vittata en la región caribe de Colombia, y se amplía su distribución. De tal modo, se avanza en el conocimiento de la especie en esta región y en el país.

\section{Materiales y métodos}

Área de estudio. La región Caribe colombiana se ubica en la porción norte del país. Colinda al oeste y al norte con el mar Caribe, y al este con Venezuela. La región incluye tradicionalmente siete unidades administrativas (departamentos): Atlántico, Bolívar, Cesar, Córdoba, La Guajira, Magdalena y Sucre. No obstante, para los fines del presente estudio, se incluyó el departamento de Antioquia, dada su amplia proporción, que corresponde a dicha región. A pesar de que geográficamente el departamento del Chocó incluye una pequeña parte dentro de la región, este departamento es tradicionalmente incluido como parte de la región Pacífico del país. Históricamente, la región ha sufrido un largo proceso de transformación. Allí, la mayoría del territorio ha sido utilizado en actividades productivas (González-Maya et al. 2013) y actualmente cuenta con pocos relictos de cobertura natural, la mayoría de los cuales están aislados.

Métodos. Se buscó toda la información disponible de la especie en el país; se incluyó la consulta de colecciones de museos nacionales e internacionales en la plataforma en línea de The Global Biodiversity Information Facility (GBIF 2015), así como 97 centros de documentación del país, entre los que se encuentran Universidades, ONG y Corporaciones Autónomas Regionales. También se realizó una búsqueda general en internet. La búsqueda simple incluyó la revisión de todos los documentos que mencionan la especie en cualquiera de sus apartados.

Adicionalmente, se obtuvo registros de campo provenientes de proyectos desarrollados por ProCAT Colombia en la región Caribe entre 2009 y 2012, en los que se usó tres metodologías complementarias: cámaras-trampa, entrevistas y transectos. Cada una de las etapas de campo se llevó a cabo en diferentes proyectos, todos enfocados en el levantamiento de información ecológica de mamíferos como herramienta de planificación para la conservación. De la base de registros de ProCAT, se extrajeron todos los registros de la especie en la región, con su respectiva coordenada geográfica. Asimismo, se incluyeron registros recientes obtenidos del trabajo de campo realizado en el departamento de Córdoba por investigadores de la Universidad de Córdoba y por la Unidad de Parques Nacionales Naturales (citados de aquí en adelante como fuente González-Maya et al., datos sin publicar).

Una vez recopilados los registros, se depuró la base de datos para obtener sólo aquéllos con información sobre la localidad y tipo de registro, en especial aquéllos con información geográfica precisa. Los registros fueron proyectados espacialmente y analizados frente a la información de 
distribución disponible en la Lista Roja de Especies Amenazadas de IUCN (Cuarón et al. 2008). Con los puntos finales obtenidos, se estimó el mínimo polígono convexo como una aproximación a su extensión de ocurrencia (i. e., extent of occurrence; Balaguera-Reina et al. 2009), de acuerdo con los lineamientos de (IUCN 2012). Se estimó el área de extensión del área de distribución aproximada de la especie respecto de la distribución previamente estimada (Cuarón et al. 2008).

\section{Resultados}

Se encontraron 161 documentos relacionados con Galictis vittata, de los cuales sólo 11 fueron específicos de la región Caribe de Colombia, con información georreferenciada sobre la presencia de la especie. Además de 78 registros en el Caribe colombiano. Hubo registros confiables distribuidos en los departamentos de Cesar, Magdalena, Sucre y Córdoba, así como registros de baja confiabilidad en los departamentos de Antioquia, La Guajira y Bolívar (Tabla 1). De éstos, $74.4 \%$ corresponde a menciones en entrevistas; $16.6 \%$, a observaciones directas; $5.13 \%$, a colectas, y 3.85 $\%$, a fotografías de cámaras trampa. Del total, el $91.03 \%$ de los registros fueron considerados de baja confiabilidad, mientras que el restante $8.97 \%$, de alta confiabilidad (Tabla 1; Figura 1). Los datos de observaciones en el departamento de Antioquia fueron considerados de baja confiabilidad dado que, a pesar de estar incluidos en GBIF, no se hallan respaldados por especímenes en colecciones ni por documentos de soporte (Corporación Autónoma Regional del Centro de Antioquia 2009). Los últimos registros confiables de la especie, sin incluir los nuevos registros aquí proporcionados, datan de 1949 y están basados, por un lado, en un espécimen colectado por Hershkovitz en el departamento de Sucre, que se encuentra depositado en la colección de The Field Museum of Natural History (FMHN), y por otro lado, en una observación de 2010 obtenida en un informe técnico de monitoreo de fauna de la Mina de Cerro Matoso. De los nuevos registros, el más reciente fue de 2012, mediante cámaras-trampa, en el Parque Nacional Natural Tayrona (Figura 2).

Los nuevos registros de distribución obtenidos, que incluyen en total 54 registros (48 de entrevistas, tres fotografías y tres observaciones; Figura 3) expanden la distribución conocida de la especie, de acuerdo con (Solari et al. 2013), a los departamentos de Córdoba, La Guajira y Cesar. Asimismo, esta nueva recopilación expande la distribución conocida en el Caribe colombiano y en el país, en aproximadamente $162 \mathrm{~km}$ lineales hacia el sur, y en aproximadamente $36,500 \mathrm{~km}^{2}$ (Figura 1).

\section{Discusión}

Los estudios relacionados con pequeños carnívoros en Colombia son escasos. En general, es poca la información existente relacionada con aspectos básicos como su distribución, ecología o estado de conservación (González-Maya et al. 2011; Suárez-Castro y Ramírez-Chaves 2015). En el país, el Caribe es una de las regiones con menor información sobre el grupo, a pesar de algunos esfuerzos recientes por documentar su biodiversidad (González-Maya et al. 2011).

El grisón ( $G$. vittata) es una de las especies de pequeños carnívoros con mayor distribución en el país; sin embargo, es al mismo tiempo una de las menos conocidas (González-Maya et al. 2011; Suárez-Castro y Ramírez-Chaves 2015; Calderón-Capote et al. 2015). Los nuevos resultados obtenidos en este trabajo aumentan significativamente la distribución conocida de la especie en el Caribe colombiano. A pesar de que las tres listas de especies de mamíferos colombianos indican la presencia de G. vittata en el Caribe, y en específico en los departamentos de Antioquia, Magdalena y Sucre (Cuervo et al. 1986; Alberico et al. 2000; Solari et al. 2013), los registros aquí presentados aumentan considerablemente la distribución de la especie en la región y en el país; además, proveen la primera recopilación de registros con algún tipo de respaldo en la región. 
Los registros obtenidos también amplían la distribución de la especie en Colombia. Se incluye registros en tres departamentos previamente no considerados en su distribución conocida (Solari et al. 2013), así como espacialmente extienden la distribución hacia el sur de la distribución propuesta por (Cuarón et al. 2008).

Es importante destacar que los registros de alta confiabilidad de la especie se obtuvieron en áreas con amplia cobertura de cuerpos de agua, concordante con el planteamiento de que la especie está fuertemente asociada a cuerpos de agua y a bosques con alta cobertura vegetal

Tabla 1. Registros confirmados de Galictis vittata en el Caribe colombiano. Tipo (TI) dentro de esta columna: observados (Ob), entrevista (En), fotos (Fo), especímen (Es). Fuente de la informacion (Ft)

\begin{tabular}{|c|c|c|c|c|c|c|c|c|c|}
\hline No. & Departamento & Municipio & Localidad & Lat. & Long. & Tipo & Año & Voucher & $\mathbf{F t}$ \\
\hline 1 & & Amalfi & Amalfi & 6.91 & -75.07 & $\mathrm{Ob}$ & 2009 & & 2 \\
\hline 2 & & Anorí & Anorí & 7.07 & -75.15 & $\mathrm{Ob}$ & 2009 & & 2 \\
\hline 3 & & Cáceres & Cáceres & 7.58 & -75.35 & $\mathrm{Ob}$ & 2009 & & 2 \\
\hline 4 & & Caucacia & Caucacia & 7.99 & -75.2 & $\mathrm{Ob}$ & 2009 & & 2 \\
\hline 5 & Antioquia & Andes & Andes & 5.66 & -75.88 & $\mathrm{Ob}$ & 2009 & & 2 \\
\hline 6 & & Ituango & Vereda Pio XII & 7.18 & -75.77 & $\mathrm{Ob}$ & 2009 & & 2 \\
\hline 7 & & Puerto Berrío & La Floresta & 6.5 & -74.71 & $\mathrm{Ob}$ & 2009 & & 2 \\
\hline 8 & & Puerto Nare & Puerto Nare & 6.18 & -74.58 & $\mathrm{Ob}$ & 2009 & & 2 \\
\hline 9 & & Zaragoza & Zaragoza & 7.5 & -74.87 & $\mathrm{Ob}$ & 2009 & & 2 \\
\hline 10 & & San Onofre & La Barce & 9.9828 & -75.5397 & En & 2010 & & 1 \\
\hline 11 & & San Onofre & San Antonio & 10.0138 & -75.5142 & En & 2010 & & 1 \\
\hline 12 & & San Juan de Nepomuceno & Corralito & 9.927 & -74.9765 & En & 2010 & & 1 \\
\hline 13 & & Arjona & Sincerín & 10.1629 & -75.2983 & En & 2010 & & 1 \\
\hline 14 & & San Juan Nepomuceno & Haya & 9.956 & -75.1883 & En & 2010 & & 1 \\
\hline 15 & & Maria la Baja & El Puerto & 10.0061 & -75.3186 & En & 2010 & & 1 \\
\hline 16 & & Maria la Baja & Maria la Baja & 9.9697 & -75.2916 & En & 2010 & & 1 \\
\hline 17 & Bolívar & Maria la Baja & El Sena & 9.9674 & -75.2888 & En & 2010 & & 1 \\
\hline 18 & & San Juan de Nepomuceno & San Juan Nepomuceno & 9.9507 & -75.0818 & En & 2010 & & 1 \\
\hline 19 & & San Juan de Nepomuceno & Las Porqueras & 9.9258 & -75.0113 & En & 2010 & & 1 \\
\hline 20 & & San Juan de Nepomuceno & San Juan Nepomuceno 2 & 9.9507 & -75.0818 & En & 2010 & & 1 \\
\hline 21 & & Maria la Baja & San Pablo & 10.0525 & -75.2699 & En & 2010 & & 1 \\
\hline 22 & & San Jacinto & San Jacinto & 9.8288 & -75.1232 & En & 2010 & & 1 \\
\hline 23 & & María la Baja & San Pablo & 10.0531 & -75.2688 & En & 2010 & & 1 \\
\hline 24 & & María la Baja & San Pablo 2 & 10.0519 & -75.2692 & En & 2010 & & 1 \\
\hline 25 & & Valledupar & El Algodón & 10.606 & -73.3116 & En & 2010 & & 1 \\
\hline 26 & & Valledupar & La Mina & 10.7028 & -73.2867 & En & 2010 & & 1 \\
\hline 27 & & Valledupar & La Sierrita & 10.244 & -73.5494 & En & 2010 & & 1 \\
\hline 28 & & Bosconia & Las Cumbres & 9.9866 & -73.8611 & En & 2010 & & 1 \\
\hline 29 & & Valledupar & Las Mercedes & 10.0698 & -73.7779 & En & 2010 & & 1 \\
\hline 30 & Cesar & Valledupar & Río Seco & 10.6078 & -73.226 & En & 2010 & & 1 \\
\hline 31 & & El Copey & Nueva idea & 10.1042 & -73.8175 & En & 2010 & & 1 \\
\hline 32 & & El Copey & Villa Rita & 10.095 & -73.8335 & En & 2010 & & 1 \\
\hline 33 & & Agustin Codazzi & Sicarare & 9.8792 & -73.4601 & Es & 1943 & USNM 281481 & 2 \\
\hline 34 & & Valledupar & Santa Monica & 10.5033 & -73.2711 & En & 2010 & & 1 \\
\hline 35 & & El Copey & Adan & 10.1904 & -73.8703 & En & 2010 & & 1 \\
\hline
\end{tabular}


Tabla 1. continuación

\begin{tabular}{|c|c|c|c|c|c|c|c|c|}
\hline 36 & & Ayapel & Caño canchila (Los Pájaros) & 8.3263 & -74.8698 & En & 2012 & \\
\hline 37 & & Buenavista & Betanci-Guacamayas & 8.1878 & -75.5349 & En & 2005 & \\
\hline 38 & & Canalete & Hacienda Chimborazo & 8.7411 & -76.3211 & En & 2006 & \\
\hline 39 & & Lorica & Cienaga de Pantano Bonito & 9.1639 & -72.8967 & En & 2004 & \\
\hline 40 & & Lorica & Finca Nueva Colombia & 9.1207 & -75.8399 & En & 2002 & \\
\hline 41 & & Los Cordobas & Reserva For. Campo Alegre & 8.7583 & -76.3256 & En & 2006 & \\
\hline 42 & & Montelibano & Ecoparque & 7.992 & -75.4071 & $\mathrm{Ob}$ & 2008 & \\
\hline 43 & & Montelibano & Cerro Matoso & 7.9128 & -75.5448 & En & 2012 & \\
\hline 44 & & Puerto Libertador & Cerro Matoso 2 & 7.9182 & -75.5707 & $\mathrm{Ob}$ & 2010 & \\
\hline 45 & & Montelibano & Las Parcelas & 7.988 & -75.5135 & En & 2012 & \\
\hline 46 & & Montería & Ciénaga de Betancí & 8.3789 & -75.8319 & En & 2004 & \\
\hline 47 & & Montería & Ciénaga de Martinica & 8.7247 & -75.9958 & En & 2004 & \\
\hline 48 & & Montería & Agua viva & 8.5199 & -76.195 & En & 2008 & \\
\hline 49 & & Montería & Universidad de Córdoba & 8.7911 & -75.8648 & $\mathrm{Ob}$ & 2011 & \\
\hline 50 & & Montería & Barrio La Castellana & 8.7675 & -75.8653 & $\mathrm{Ob}$ & 2011 & \\
\hline 51 & & Montería & Universidad de Córdoba & 8.7931 & -75.8615 & Fo & 2010 & \\
\hline 52 & Córdoba & Moñitos & Reserva Natural Viento Solar & 9.1371 & -76.1838 & En & 2006 & \\
\hline 53 & & Pueblo Nuevo & NoData & 8.3411 & -75.3244 & En & 2005 & \\
\hline 54 & & Puerto Escondido & Finca Leticia & 9.0936 & -76.205 & En & 2000 & \\
\hline 55 & & Puerto Libertador & Alto Río Uré & 7.6369 & -75.6419 & En & 2000 & \\
\hline 56 & & San Pelayo & Ciénaga La Pacha & 9.0402 & -75.8503 & En & 2004 & \\
\hline 57 & & Tierralta & Quebrada Tai & 7.97 & -76.1625 & En & 2000 & \\
\hline 58 & & Tierralta & El Silencio & 6.0118 & -76.0589 & En & 2009 & \\
\hline 59 & & Montería & Hacienda Currayao & 8.3947 & -75.9535 & En & 2010 & \\
\hline 60 & & Tierralta & Finca Walterra & 8.2777 & -76.0346 & En & 2009 & \\
\hline 61 & & Tierralta & Hacienda Monaco & 8.3259 & -75.9854 & En & 2009 & \\
\hline 62 & & Montelibano & Vereda El Diamante (Cerro Murrucucú) & 7.9844 & -75.9667 & En & 2008 & \\
\hline 63 & & Montelibano & Vereda La Chica (Cerro Murrucucú) & 8.0064 & -75.9783 & En & 2008 & \\
\hline 64 & & Montelibano & Cerro Murrucucú & 7.8739 & -75.98 & En & 2008 & \\
\hline 65 & & Montelibano & Vereda EI Venado (Cerro Murrucucú) & 7.8778 & -75.9378 & En & 2008 & \\
\hline 66 & & Montelibano & San Pedro (Cerro Murrucucú) & 7.9331 & -75.9642 & En & 2008 & \\
\hline 67 & & Montería & Hacienda Las Tangas & 8.3375 & -76.0714 & En & 2009 & \\
\hline 68 & & Valencia & Hacienda Jaraguay & 8.3012 & -76.0655 & En & 2008 & \\
\hline 69 & \multirow{4}{*}{ La Guajira } & Maicao & Los Paraujanos & 11.1895 & -72.3525 & En & 2010 & \\
\hline 70 & & San Juan del Cesar & Corraleja & 10.7356 & -72.8522 & En & 2010 & \\
\hline 71 & & Albania & Nueva América & 11.2151 & -72.5812 & En & 2010 & \\
\hline 72 & & Riohacha & Tomarrazón & 11.1266 & -72.9256 & En & 2010 & \\
\hline 73 & \multirow{4}{*}{ Magdalena } & Sabanas de San Ángel & Pueblo Barrios & 10.1748 & 74.3272 & En & 2013 & \\
\hline 74 & & Santa Marta & El Coquito (Parque Nacional Tayrona) & 11.3237 & -73.9687 & Fo & 2012 & \\
\hline 75 & & Santa Marta & Arrecifes (Parque Nacional Tayrona) & 11.3065 & -73.9639 & Fo & 2012 & \\
\hline 76 & & Sitio Nuevo & La Trinidad & 10.851 & -74.6188 & Es & Sd & USNM 544420 \\
\hline 77 & \multirow{2}{*}{ Sucre } & Colosó & Ricaurte & 9.5 & -75.35 & Es & Sd & IAvH-M-5547 \\
\hline 78 & & Colosó & Ricaurte 2 & 9.5 & -75.35 & Es & 1949 & FMNH 68906 \\
\hline
\end{tabular}




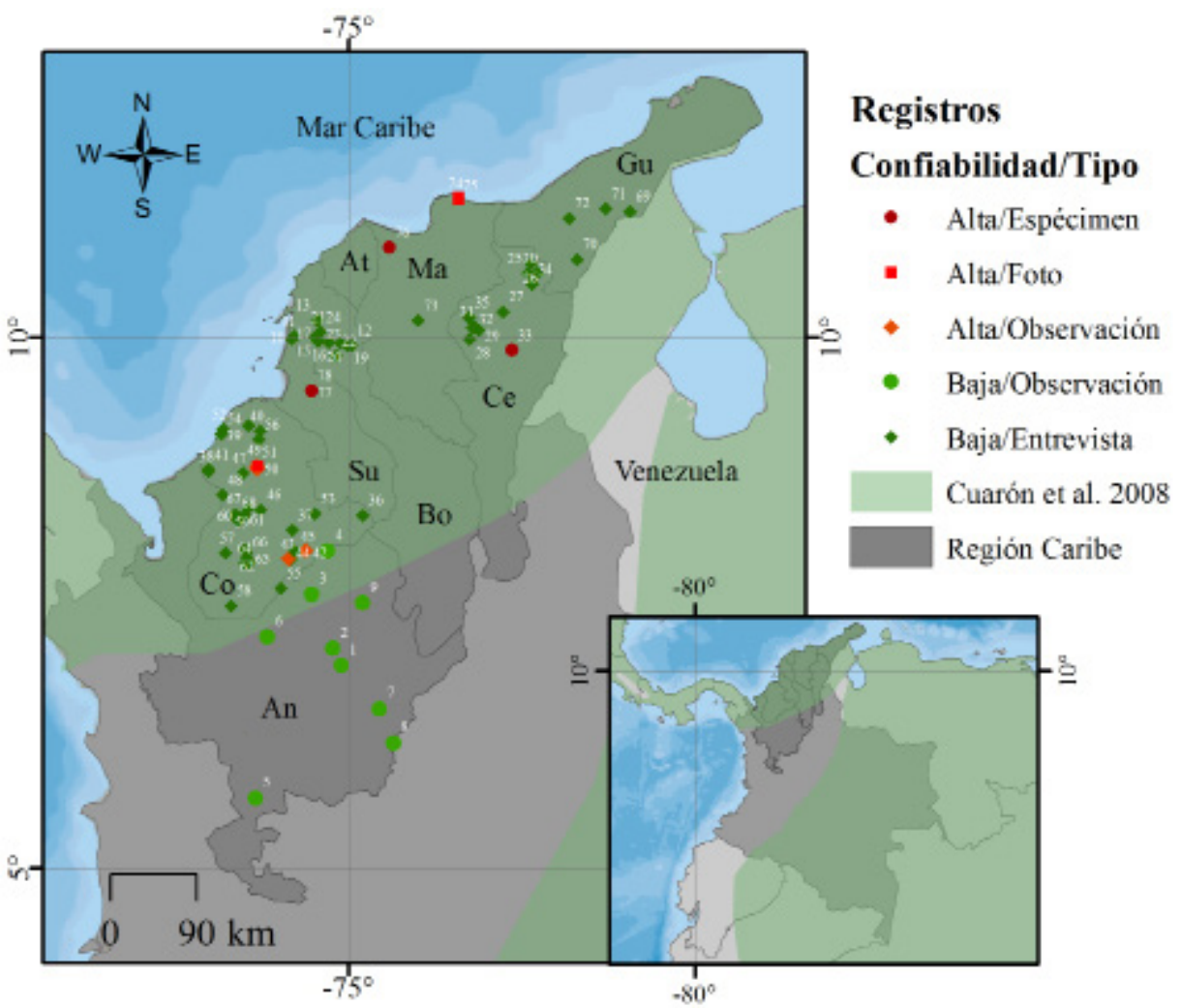

Figura 1. Registros de Galictis vittata en el Caribe Colombiano, discriminados según su confiabilidad y tipo de registro (los números en blanco indican el registro de acuerdo con la Tabla 1) y distribución según la Lista Roja de Especies Amenazadas de UICN (Cuarón et al. 2008). Departamentos: An: Antioquia, At: Atlántico, Bo: Bolívar, Ce: Cesar, Co: Córdoba, Gu: La Guajira, Ma: Magdalena y Su: Sucre.

(Yensen y Tarifa 2003; Cuarón et al. 2008). Es también de destacar que la mayoría de su distribución actual está siendo alterada por las diversas presiones antrópicas. Por lo tanto, es importante realizar estudios que evalúen la tolerancia de la especie a las presiones ejercidas en su distribución natural, y de esta manera, definir una real categoría de amenaza para la especie.

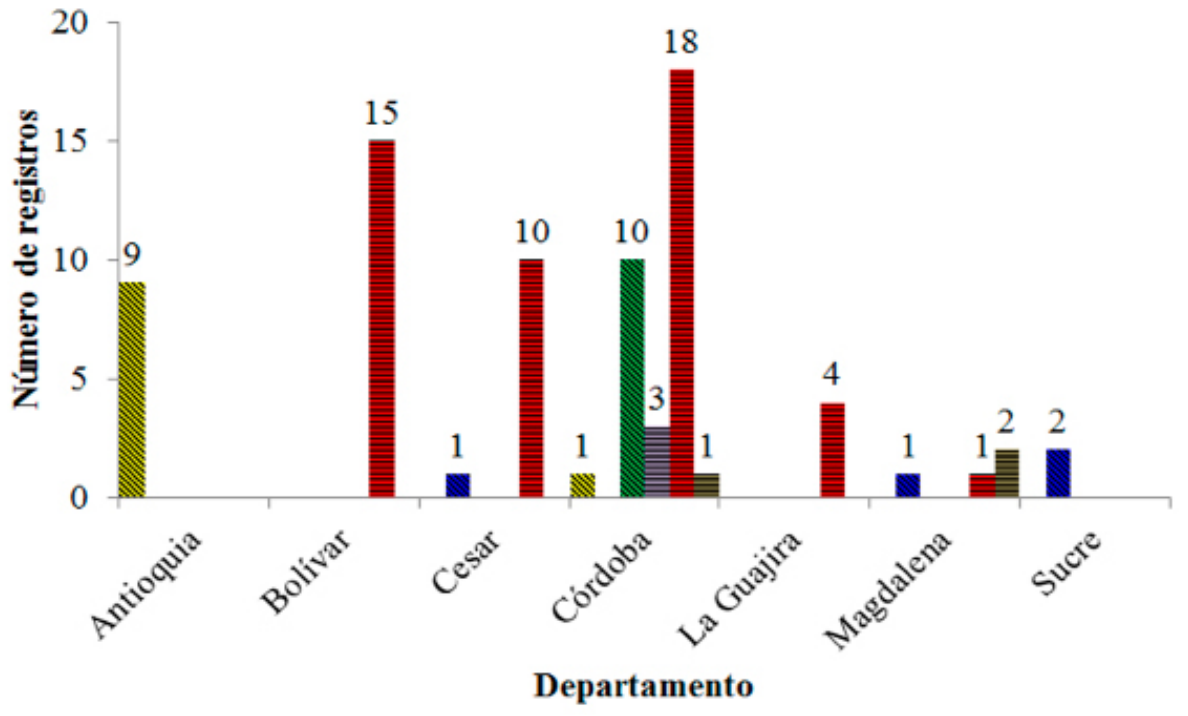

Figura 2. Distribución por departamentos de los registros, discriminados por tipo, de Galictis vittata en el Caribe Colombiano. Las barras con tramado en diagonal indican registros obtenidos de la revisión de literatura, divididos en Observaciones (amarillo), Especímenes (azul) y entrevitas (verde); barras con tramado horizontal indican registros nuevos obtenidos en esfuerzos de campo reciente por observaciones (gris), entrevistas (rojo) y fotos (ocre). Los números sobre cada barra indican el número de registros por tipo. 


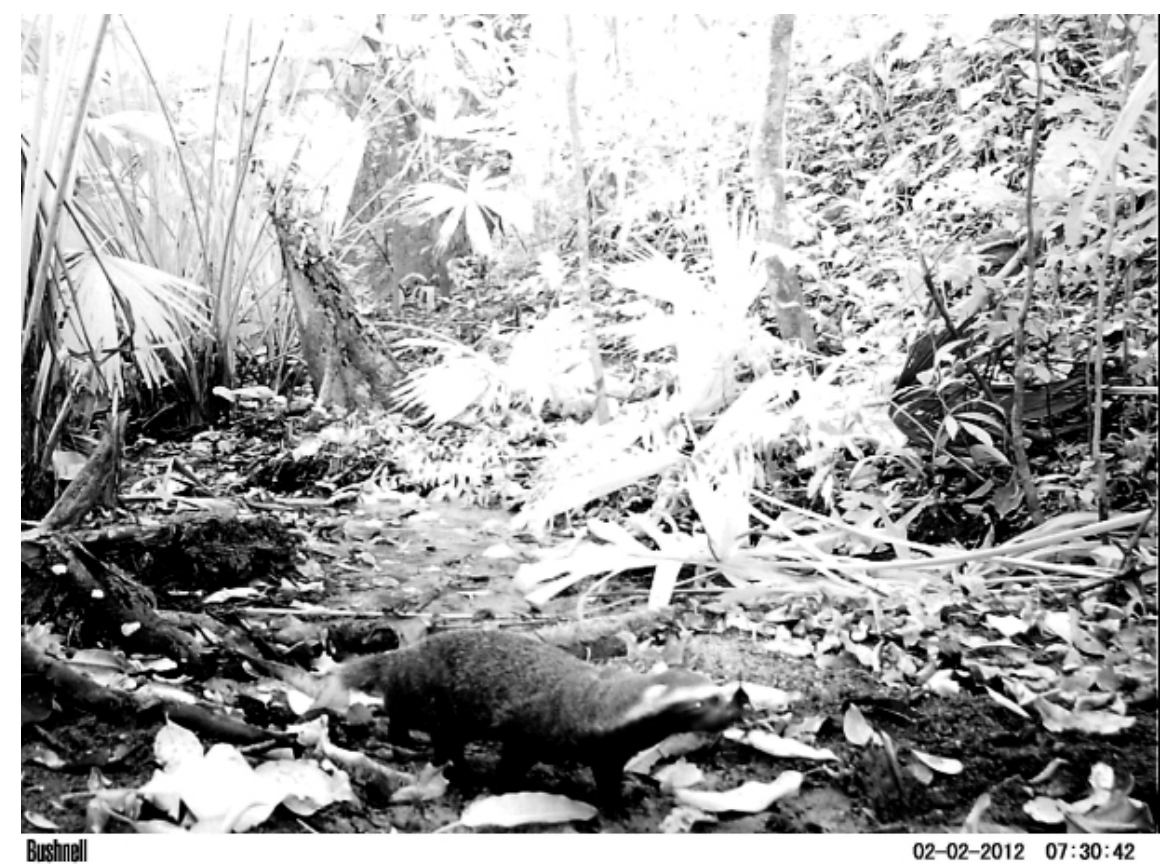

Figura 3. Último registro confirmado de Galictis vittata en el Caribe colombiano, obtenido en febrero de 2012 en el Parque Nacional Natural Tayrona, Magdalena, Colombia.

Dado el considerable vacío de conocimiento sobre la especie, esta nueva información aporta conocimiento sobre la distribución de la misma, y puede servir como base para análisis posteriores que involucren aspectos de su ecología y estado de conservación. Teniendo en cuenta los fuertes procesos de trasformación de los ecosistemas naturales en el país, y en especial en el Caribe colombiano (GonzálezMaya et al. 2013), contar con información actualizada y completa de la distribución de las especies es fundamental como base para planificar su conservación.

\section{Agradecimientos}

Agradecemos a la fundación ProCAT Colombia por su continuo apoyo durante el proceso de este trabajo. Agradecemos también a Parques Nacionales por su colaboración en campo. Un agradecimiento especial al editor y a los dos revisores anónimos por sus aportes, que mejoraron considerablemente la calidad y claridad del manuscrito.

\section{Literatura citada}

Alberico, M., A. Cadena, J. Hernández-Camacho, y Y. Muñoz-Saba. 2000. Mamíferos (Synapsida: Theria) de Colombia. Biota Colombiana 1: 3-75.

Asprilla-Perea, J., J. E. Serna-Agudelo, y Y. Palacios-Asprilla. 2013. Diagnóstico sobre el decomiso de fauna silvestre en el departamento del Chocó (Pacífico Norte colombiano). Revista UDCA Actualidad \& Divulgación Científica 16:175-184.

Balaguera-Reina, S. A., A. Cepeda, D. A. Zárrate-charry, y J. F. González-Maya. 2009. The state of knowledge of Western Mountain Coati Nasuella olivacea in Colombia, and extent of occurrence in the Northern Andes. Small Carnivore Conservation 41:35-40. 
Calderón-Capote, M. C., A. V. Rojas-Rojas, C. Cárdenas-González, y A. Pardo-Ramírez. 2015. Familia Mustelidae. Pp. 110-133 en Los carnívoros terrestres y semiacuáticos continentales de Colombia: Guía de Campo (Suárez-Castro, A. F., y H. E. RamírezChaves, eds.). Universidad Nacional de Colombia. Bogotá, Colombia.

Corporación Autónoma Regional del Centro de Antioquia. 2009. Inventario de fauna presente en la jurisdicción de Corantioquia, Versión 2. Corporación Autónoma Regional del Centro de Antioquia. <ipt.sibcolombia.net/sib/resource.do?r=corantioquia-2008fauna>.

Cuarón, A. D., Reid, F., y K. Helgen. 2008. Galictis vittata. IUCN Red List of Threatened Species, Version 2011.2. <WWw.iucnredlist.org $>$.

Cuervo Díaz, A., J. I. Hernández Camacho, y A. Cadena. 1986. Lista actualizada de los mamíferos de Colombia: anotaciones sobre su distribución. Caldasia 15:471-501.

GBif - The Global Biodiversity Information Facility. 2015. GBIF Backbone Taxonomy, Galictis vittata. The Global Biodiversity Information Facility. http://www.gbif.org/ species/5218906.

González-Maya, J. F., A. A. Cepeda-Mercado, J. L. Belant, D. A. Zárrate-Charry, S. A. BalagueraReina, y A. Rodríguez-Bolaños. 2011. Research priorities for the small carnivores of Colombia. Small Carnivore Conservation 44:7-13.

González-Maya, J. F., J. F. Romero-Rendón, D. A. Zárrate-Charry, C. Castaño-Uribe, M. González, L. R. Víquez-R, y A. Arias-Alzate. 2013. Evaluación geográfica y prioridades de conservación de hábitat para felinos en el Caribe colombiano. Pp. 77-87 en Plan de Conservación de Felinos del Caribe Colombiano: Los felinos y su papel en la planificación regional integral basada en especies clave (Castaño-Uribe, C., J. F. González-Maya, D. A. Zárrate-Charry, C. Ange-Jaramillo, y I. M. Vela-Vargas, eds.). Fundación Herencia Ambiental Caribe, ProCAT Colombia. The Sierra to Sea Institute. Santa Marta, Colombia.

IUCN. 2012. Guidelines for application of IUCN Red List criteria at regional and national levels. IUCN Species Survival Commission, Gland, Switzerland.

Muñoz-Saba, Y., y M. Alberico. 2004. Mamíferos en el Chocó biogeográfico. Pp. 559-598 en Diversidad Biótica IV. El chocó Biogeográfico/Costa Pacífica (Rangel-Ch., J. O., ed.). Universidad Nacional de Colombia, Instituto de Ciencias Naturales, Conservación Internacional. Bogotá, Colombia.

Solari, S., Y. Muñoz-Saba, J. V. Rodríguez-Mahecha, T. Defler, H. E. Ramírez-Chaves, y F. Trujillo. 2013. Riqueza, endemismo y conservación de los mamíferos de Colombia. Mastozoología Neotropical 20:301-365.

Suárez-Castro, A. F., y H Ramírez-Chávez. 2015. Introducción. Pp. 18-21 en Los carnívoros terrestres y semiacuáticos continentales de Colombia: Guía de Campo (Suárez-Castro, A. F., y H. E. Ramírez-Chaves, eds.). Universidad Nacional de Colombia. Bogotá, Colombia.

Yensen, E., y T. Tarifa. 2003. Galictis vittata. Mammalian Species 727:1-8.

Summited: June 9, 2015

Review: August 22, 2015

Accepted: October 6, 2015

Associated editor: Guillermo D'Elia 